\title{
Algunas cuestiones de interés sobre el poblamiento del continente europeo durante el Pleistoceno Medio
}

\author{
juan Antonio Martos Romero *
}

"Nuevas islas, nuevas tierras, nuevos mares, nuevos pueblos, y lo que es mejor, un nuevo ciclo y nuevas estrellas.”

Citado por Joaquim de Carvalho, "Estudos sobre a cultura portuguesa do século XV|», I (Coimbra, 1947), 42.

A nuestro modo de ver ${ }^{* *}$, existen desde muy pronto dos temas en la investigación del Pleistoceno Inferior y Medio que resultan de especial atracción para los arqueólogos interesados por este marco cronológico y geográfico, y que tienen la envidiable capacidad de disparar sus mentes y de dar lugar a teorias e hipótesis unas veces fascinantes, otras provocativas, las más contradictorias, pero todas ellas controvertidas. Ambos temas nos incumben muy de cerca como únicos representantes del género Homo en la actualidad y como europeos que somos. Sin duda es por ello por lo que resultan tan atractivos y sujetos a continuos debates, discusiones y visiones contrapuestas y a veces irreconciliables, aunque siempre constructivas para la ciencia de la arqueología. Por un lado, nos referimos a la cuestión del origen del género Homo, su antigüedad como tal y la configuración de su rama filogenética. Por otro lado, estamos ante el no menos complejo interrogante del primer poblamiento de Europa durante el Pleistoceno. En el presente artículo pretendemos abordar algunas de las cuestiones que más preocupan e interesan en la UNED.

* Becario Predoctoral del Departamento de Prehistoria e Historia Antigua de la

** Quiero dedicar este trabajo al Dr. Sergio Ripoll López y a mis compañeros de equipo, por el incondicional apoyo que desde siempre me han brindado y la sincera amistad que me une a ellos. 
actualidad a los investigadores más reconocidos a nivel mundial en los estudios ligados al Pleistoceno, acerca de la ocupación efectiva del continente europeo por los humanos salidos de Africa. Nuestro objetivo se centra en reunir y desarrollar minimamente algunas de esas cuestiones. Su tratamiento han dado lugar $u$ origen en los últimos años a una serie de puntos de interés, significativos por los planteamientos e implicaciones que vienen a desarrollar y que expondremos articulados en cuatro grandes bloques. Estos bloques se perfilan como el perfecto encabezamiento para discutir las cuestiones que plantea en la actualidad el tema que aquí recogemos; la cronología, el medio, las industrias y las estrategias de subsistencia de estos primeros habitantes de Europa.

La elección del tema responde a un interés personal por el mismo dentro de mi línea de investigación actual, muy ligada a la arqueología del Pleistoceno Medio, y al hecho de que en un momento como el presente, donde el concepto de Europa y de lo "europeo" es tan pujante y a su vez controvertido, es de interés volver la mirada siquiera sea un instante hacia los que fueron los más antiguos europeos.

Antes de comenzar daremos un salto, sin duda vertiginoso, obviando toda una serie de preguntas que se nos plantearían de forma inicial al enfocar el estudio del primer poblamiento del continente europeo. ¿Cuál fue el punto de origen, y el camino que siguieron los pobladores y/o descubridores del suelo europeo?, ¿quiénes eran esos primeros exploradores?, ¿cuánto tiempo duró el viaje?, ¿qué fue lo que lo provocó?, ¿qué avatares acompañaron una empresa de tal calibre? y ¿cuáles fueron las rutas de desplazamiento hacia Europa? Interrogantes todos ellos de gran interés y problemática y de los que se puede encontrar una buena síntesis en el trabajo de Rolland (1992).

Nuestra exposición arranca cuando todos esos interrogantes que hemos planteado ya se han sucedido en el tiempo y en el espacio. Tiene su punto de partida en la revisión de las evidencias que se manejan como las primeras pruebas materiales de la presencia efectiva del género humano en el continente europeo.

\section{LA CUESTIÓN CRONOLÓGICA}

La cronología del primer poblamiento de Europa se ha planteado como un tema de interés prioritario en las investigaciones arqueológicas; buscar y encontrar la prueba más remota de la llegada de los primeros seres humanos al continente ha ocupado el tiempo y las energías de muchas generaciones de paleolitistas. Sin embargo, pese a todo ese enorme "input" de esfuerzo material e intelectual, el resultado nos pare- 
Algunas cuestiones de interés sobre el poblamiento del continente europeo...

ceria muy escaso a la luz de la enorme controversia a la que están sujetos todos los interrogantes y respuestas que plantea dicha cuestión. La controversia que rodea la fecha del poblamiento del continente europeo tiene su raiz en las evidencias que se manejan como pruebas de una ocupación antigua de Europa por los erectus ${ }^{1}$.

¿De dónde provienen esas evidencias? Dos son los puntos de referencia clave:

a) Determinar el carácter humano o no humano (natural) de los artefactos y/o conjuntos líticos registrados.

b) Definir la posición cronológica de esos artefactos y/o conjuntos líticos registrados.

Es decir, determinar la autoria, la participación del ser humano en la fabricación de las evidencias líticas y en segundo lugar establecer su antigüedad en el tiempo.

Estos dos puntos resultan sumamente complejos de conseguir cuando se aplican a materiales líticos que se pretenden remontar por algunos investigadores a fechas del Pleistoceno Inferior. La posible existencia y búsqueda de artefactos de una gran antigüedad en la escala del Pleistoceno, que hicieran retroceder en el tiempo la llegada de los primeros europeos, los futuros "civilizadores del mundo", dio lugar a una extensa literatura unas veces científica y otras, más propias de un género como el de la ciencia ficción. Desde principios de siglo prehistoriadores y geólogos se preocuparon por determinar si los artefactos que encontraban en las terrazas más altas de los ríos europeos estaban realizados por humanos o si por el contrario su morfologia era obra de la naturaleza: los denominados "eolitos".

Discernir el carácter humano o no de artefactos líticos tan remotos en el tiempo resulta enormemente difícil. Un ejemplo de trabajo en este sentido es la obra de J. Desmond Clark (1958) con algunas industrias africanas. De igual manera, dichos artefactos se vuelven aún más controvertidos debido a la propia naturaleza de los hallazgos, piezas sueltas recogidas en terrazas fluviales o conjuntos descontextualizados que carecen de cualquier posibilidad de atribución cronológica. Parece evi-

\footnotetext{
Pese a que autores como Querol (1991) consideran a los primeros pobladores del continente europeo como los representantes más arcaicos de la especie sapiens utilizando la denominación sapiens arcaicos, ante el alto grado de polimortismo que se observa en el registro fósil humano que conforma el grupo erectus en Europa, optamos aqui por la diagnosis erectus.
} 
dente pues que aquellos que se preocupan por buscar y encontrar una fecha alta para el poblamiento de Europa manejan unas evidencias (el material lítico) sujetas a una serie de controversias inevitables (la naturaleza humana o no humana del mismo y su posición cronológica).

Ante la dificultad y complejidad de trabajar con tales parámetros parece lógico proponer unas líneas de trabajo esenciales para paliar estos inconvenientes y obtener un mayor grado de fiabilidad en el estudio de las evidencias más antiguas, tal y como proponen Roebroeks y Van Kolfschoten (1994). Estos investigadores destacan dos puntos esenciales a la hora de enfrentarse a este tipo de evidencias:

a) La realización de un profundo estudio de toda la gama de condiciones naturales que rodean el sitio y que pudieran producir formas semejantes a las de los artefactos realizados por humanos.

b) La realización de un profundo estudio de las características geológicas y geomorfológicas de los sitios donde los artefactos son recogidos.

Como hemos expuesto, la fecha del primer poblamiento es el primero de los debates sometidos a una fuerte controversia. Actualmente las posiciones de los investigadores pueden resumirse en dos frentes:

a) Aquellos que defienden una cronología alta para el poblamiento de Europa. Situan sus fechas en el Pleistoceno Inferior con cronologias en torno a los dos millones de años. Bonifay y Vandermeersch (1991), Ackerman (1989) o Delson (1989) apoyan esta cronología alta.

b) Los partidarios por contra de una cronologia baja para la ocupación del continente europeo. Argumentan que las evidencias consistentes de un poblamiento efectivo de Europa sólo pueden admitirse a partir de hace 500.000 años. La mayoria de los paleolitistas se muestran de acuerdo, en la actualidad, con esta cronología de Pleistoceno Medio. Ello se debe de forma esencial a dos razones. En primer lugar, ante la falta de solidez de las evidencias presentadas con cronologias anteriores. En segundo lugar, debido a su escasez frente al número de hallazgos datados alrededor de esa fecha convencional de los 0,5 millones de años.

En un punto intermedio se situaria la postura de investigadores como Rolland (1992), que optan por una fecha cercana a un millón de años, hacia finales del Pleistoceno Inferior. Rolland argumenta su postura con yacimientos como Le Vallonet o Soleilhac en el macizo central francés, con dataciones en torno a los 0,9 millones de años. Contempla el fenómeno del poblamiento dentro de una perspectiva biogeográfica muy interesante, en la línea de los presupuestos teóricos de Turner (1982), haciendo coincidir la llegada de los primeros Homo a Europa con el evento bio- 
lógico que supone el paso del estadio Villafranquiense al Galeriense hace aproximadamente 0,97 millones de años. Rolland plantea como una posibilidad sugerente, que los representantes del género Homo hubiesen sido una más de las especies de mamiferos que vinieron a caracterizar dicho evento. De esta forma consigue dos objetivos. Una simbiosis entre los datos arqueológicos y paleoecológicos y una explicación a los motivos que llevaron a la expansión de los homínidos fuera de Africa. Sin embargo, no faltan problemas a sus propuestas, empezando por la controversia que rodea a yacimientos como Le Vallonet o Soleilhac. También podemos preguntarnos si la sustitución de la asociación faunistica del Villafranquiense fue rápida o gradual y cuál fue ese intervalo de tiempo o si en la irradiación de especies desde el continente africano todas siguieron el mismo camino.

Llegados a este punto, debemos plantearnos, ¿qué datos nos proporciona el registro arqueológico? y si, ¿abogan éstos por una cronologia alta o por una cronología baja?

\section{El Registro de fósiles humanos}

Es el primero de los apoyos con el que contamos en una revisión de las evidencias en pro y en contra de una cronologia alta o baja. Si observamos la distribución cronológica y geográfica de este registro fósil, podemos extraer dos conclusiones inmediatas:

a) Todos los fósiles humanos más antiguos de Europa pertenecen al Pleistoceno Medio. No hay ninguno anterior.

b) Los restos de fósiles humanos situados en esta cronologia de Pleistoceno Medio se encuentran repartidos prácticamente por toda Europa, a excepción de la región escandinava.

Hasta el momento presente, ninguno de los sitios que han proporcionado restos fósiles humanos: Bianche-Saint-Vaast, Bilzingsleben, Ehringsdorf, Mauer, Steinheim (Alemania); Atapuerca (España); Petralona (Grecia); Pontnewyyd, Swanscombe (Gran Bretaña); Arago, La Chaise, Fontéchevade, Grotte du Prince, Lazaret, Montmaurin, Ornag III, Vergranne (Francia); Vérstesszöllös (Hungria); Cava Pompi, Castel di Guido, Fontana Ranuccio, Venosa-Loreto, Visogliano (Italia) (Cook et al., 1982); va más allá del Pleistoceno Medio. A esta lista habria que añadir el reciente hallazgo de una tibia en el yacimiento inglés de Boxgrove (Roberts 1994) con una cronología alta, pero dentro de los parámetros de quienes defienden una cronología baja para el poblamiento de Europa situada en el Pleistoceno Medio, no más allá de 0,5 millones de años. 


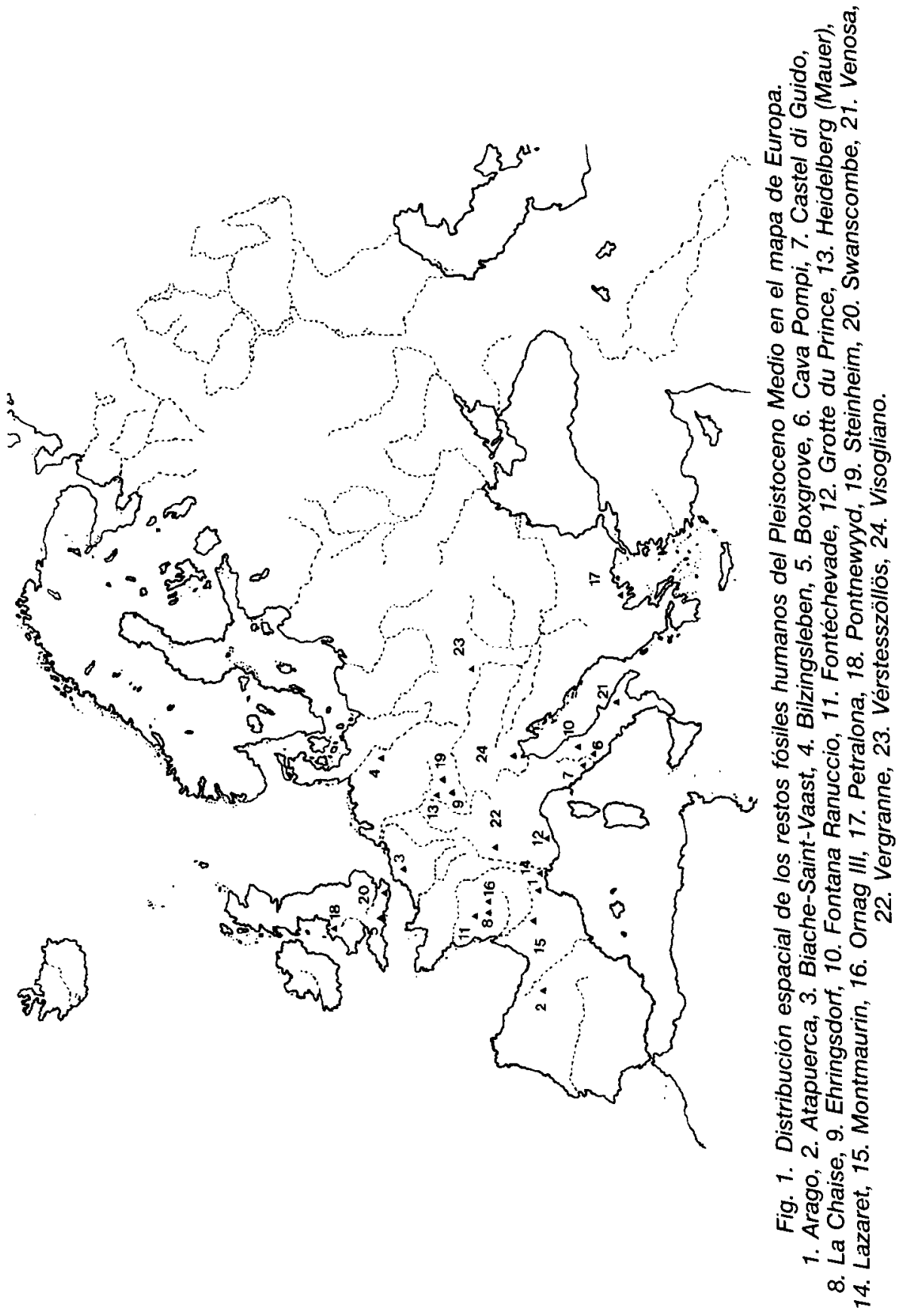


Un yacimiento que podría venir a alterar esta situación es el sitio de Venta Micena (Granada, España), donde sus excavadores han registrado un fragmento de homínido (Gibert 1985) asociados a un conjunto faunístico de características villafranquienses y datado entre 1,6 y 0,9 millones de años. Si este resto fósil perteneciese a un humano, se convertiría en el más antiguo y en el único situado en el Pleistoceno Inferior. Sin embargo, el yacimiento presenta una fuerte controversia y problemática para la comunidad científica por lo que el sitio queda sujeto todavía a un enorme interrogante y escepticismo: "The absence of stone artifacts at the site is surprinsing and cause for scepticism, especially since several hundred square meters have been excavated» (Santonja \& Villa 1990, pág. 82). Aunque es posible que durante el Congreso Internacional de Paleontología Humana a celebrar en Orce en 1995 se clarifique esta situación.

Lo que sin duda es destacable si comparamos los datos del registro fósil humano con los del registro lítico es la enorme distancia que existe entre las fechas que se atribuyen a algunas industrias y los fósiles humanos más antiguos. Distancia que supera el millón de años en algunos casos. Habría que preguntarse por las causas de esta falta de simbiosis cronológica. Es decir, ¿por qué no hay restos humanos anteriores a 0,5 millones de años? Las respuestas a esta pregunta pueden ser muchas y de muy variada indole, no obstante nosotros destacamos tres principales:

a) Un vacio en la investigación o una falta de excavaciones en sitios que favorezcan una conservación de restos de tales características.

b) Inexistencia de superficies viejas al ser el suelo de Europa un suelo muy antropizado.

c) Que Europa no fuese habitada de forma efectiva hasta el Pleistoceno Medio, como defienden los partidarios de una cronología baja.

Estamos de acuerdo con Roebroeks y Van Kolfschoten (1994) en que las dos primeras opciones podrian carecer de justificación, si alegamos la existencia de numerosos yacimientos paleontológicos con faunas del Pleistoceno Inferior.

Asi pues parece lógico concluir que la inexistencia de restos fósiles humanos con anterioridad al Pleistoceno Medio supone un apoyo para los defensores de una cronología baja para el poblamiento de Europa (fig. 1). No obstante, la discordancia cronológica con las evidencias líticas que proporcionan algunos sitios resulta tan contundente que conviene revisar tales evidencias antes de extraer las conclusiones e implicaciones que supone aceptar una cronologia baja. 
El registro de los sitios y su cronologia

Vamos a revisar brevemente los sitios que presentan conjuntos líticos con dataciones muy altas. Todos ellos están rodeados de un enorme escepticismo, y como veremos posteriormente, presentan una serie de problemas y carencias comunes que restan solidez a las interpretaciones que de ellos hacen sus excavadores ${ }^{2}$.

\section{Centro-Este}

Beroun (Fridrich 1991)

En este sitio cercano a Praga se recogieron unos ochenta artefactos en una superficie de gravas de unos dos metros cuadrados. Fridrich describió a partir de estos materiales una industria muy antigua compuesta de choppers, bifaces, protobifaces, hendedores, poliedros y subesferoides. Se definió esta industria en base a su tipologia como un Achelense inicial equiparable al africano. No obstante, especialistas como Roebroeks o Kozlowski (1992) descartan la autoria humana para esta industria, ya que las piezas están muy rodadas y la mayor parte, descrita como choppers monofaciales, presenta únicamente unos pocos negativos. No lo consideran una evidencia aceptable y optan por clasificarlos como ecofactos.

\section{Musov I (Valoch 1991)}

El sitio se localiza a unos $40 \mathrm{~km}$ al sur de Brno. Fueron recogidos cientos de choppers y chopping tools a techo de unos depósitos removidos y que se atribuyeron al Pleistoceno Inferior o Inferior Medio. Los materiales presentaban únicamente unos pocos e irregulares negativos, así como los filos muy alterados.

Estariamos en este caso ante un claro ejemplo de una selección de piezas por su supuesta morfología "primitiva" entre miles y miles de guijarros, ninguno de ellos modificados por los humanos.

2 Los datos de los sitios que reflejamos aquí están tomados esencialmente de Roebroeks \& Van Kolfschoten (1994), Santonja \& Villa (1990) y Villa (1991). 
Prezletice (Fridrich 1989)

Por su fauna que presenta el género Mymomis y con el apoyo del paleomagnetismo fue citado como un yacimiento antiguo, de finales del Pleistoceno Inferior. Excavado entre 1965 y 1985, se trata de unos depósitos cercanos a un antiguo lago. Se describen cuatro horizontes con artefactos, clasificados principalmente como productos del resto de talla. Pese a que tal y como informan Roebroeks y Van Kolfschonten los dibujos de Fridrich muestran un material que presenta abundantes negativos, producto de un trabajo de talla, así como retoques sobre piezas descritas como "protobifaces" y otros artefactos, estos mismos autores denuncian que existen claras dudas del carácter humano de tales artefactos si se observa ese mismo material fotografiado. Resulta evidente para ellos que es enormemente difícil admitir esa participación humana en su manufactura. También es muy complejo poder diferenciar entre este material, qué corresponde a un resto de talla y qué corresponde a fracturas naturales de la materia prima. Sorprende en todo caso la completa ausencia de lascas o núcleos. Tampoco se observan entre este material las trazas que pudieran denunciar un trabajo de talla, principalmente bulbos.

\section{Stránská Skála (Valoch 1987)}

Este sitio presenta una fauna muy similar a la de Prezletice. Valoch describió algunas lascas que podian sugerir una actuación humana, procedentes de la criba de depósitos excavados entre 1910 y 1945 . El sitio se presenta como muy problemático. Los nódulos de materia prima se encuentran con gran frecuencia craquelados y rotos por causas naturales. Asi fragmentados tales nódulos, es enormemente difícil discernir cuáles pudieron deberse a una acción antrópica.

Posteriormente, en la década de los ochenta, a raíz de una serie de trabajos paleontológicos se dieron a conocer nuevos hallazgos en depósitos situados en una pendiente, así como en el interior de dos cavidades de la ladera (Valoch 1987). Valoch identificó entonces una treintena de artefactos. Sin embargo, éstos no parecen mostrar pruebas claras de una realización humana. No presentan bulbos, ni claros negativos $u$ ondas de percusión. Ni los atributos de las piezas, ni el contexto donde fueron recogidas, permiten demostrar una ocupación durante el Pleistoceno Medio Antiguo en esta zona.

Así pues, existen claras dudas sobre el carácter antrópico de todos estos conjuntos. El primer sitio que se admite de forma contundente 
como una evidencia sólida de ocupación es el de Sedlesovice (Znojmo), con un útil en lasca hallado en un perfil loéssico de un suelo fósil datado en el Holstein, es decir en torno al 0,5 millones de años.

\section{Centroeuropa}

Kärlich A (Wurges 1986, Vollbrecht 1992)

Este conocido yacimiento alemán posee una amplia secuencia estratigráfica para el Pleistoceno. El nivel A está compuesto por un depósito de gravas, arenas y loess que se sitúa en el estadio 23 de la curva isotópica con una fecha de unos 900.000 años. Wurges presentó tres «pebble-tools", todos con una talla monofacial, uno de los cuales mostraba lo que podria reconocerse como negativos. Sin embargo, las dudas son razonables y Roebroeks opta por definirlos como ecofactos o en todo caso situarlos en una categoría de «incertofactos». Por otra parte, la falta de un contexto estratigráfico se añade a la problemática de estas piezas (fig. 2).

Kärlich Ba (Wurges 1986, Vollbrecht 1992)

Depósito de gravas en la facies del río Rhin, presenta alteraciones debidas a fenómenos de crioturbación entre otros. Se correlaciona con el estadio 20 de la curva isotópica, lo que lo sitúa a finales del Pleistoceno Inferior. Se presentó un posible núcleo. Una vez más, la falta de contexto estratigráfico claro compromete cualquier interpretación posterior.

Kärlich Bb (Wurges 1986, Vollbrecht 1992)

El depósito se corresponde esta vez con una superficie de gravas de la facies del río Mosela. Se coloca inmediatamente después del evento paleomagnético del Matuyama-Brunhes. Se recogieron ocho piezas en cuarcita en un área de aproximadamente mil seiscientos metros cuadrados. Presenta problemas de carácter estratigráfico, ya que el conjunto reúne piezas tanto del techo como de la base de este paquete de gravas. Habria que tener en cuenta fenómenos de deslizamiento y solifluxión. Algunas piezas están muy rodadas mientras que otras lo están menos. Esta falta de homogeneidad viene a desacreditar el hallazgo. Bosinski describe las piezas como ligeramente rodadas y las clasifica 

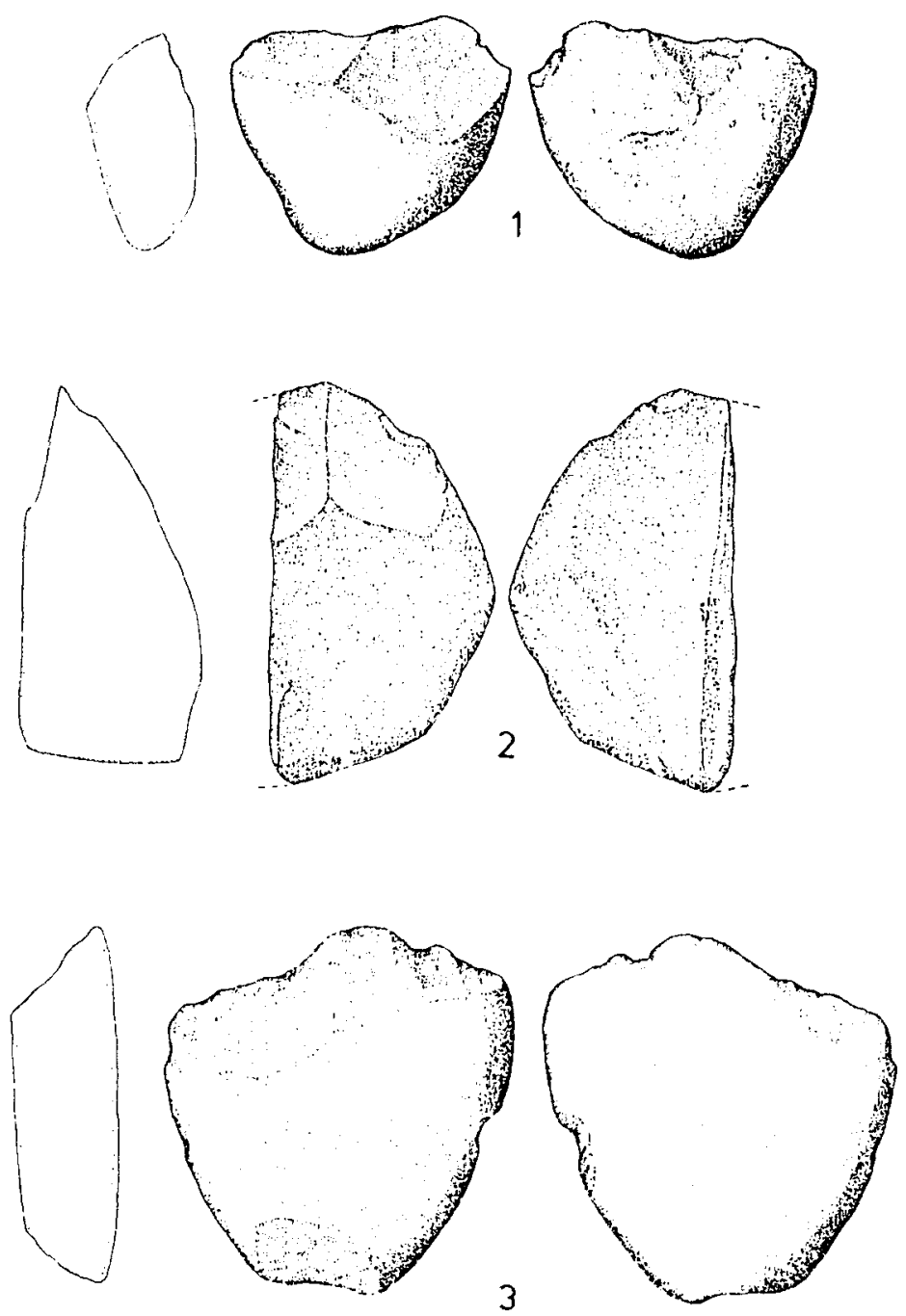

Fig. 2. Kärlich A, Pebble tools. (Wurges \& Vollbrecht). 
como dos núcleos, cuatro lascas, una raedera y un protobifaz. Para Roebroeks las características del hallazgo implican que hubo una seleccion de piezas y que éstas representan un número ínfimo comparado con el posible total si se observa el contexto donde fueron recogidas. En segundo lugar, queda claro que la selección se basó en su morfología "primitiva". El contexto de su hallazgo hace dudar de su carácter antrópico.

La primera evidencia sólida para esta zona es Kärlich G, entre los estadios 11 y 13, es decir entre 362.000 y 524.000 años. En esta misma situación se sitúan yacimientos como Mauer o Miesenheim. La fecha del Pleistoceno Medio se refuerza con la presencia en estos yacimientos del género Arvicola, concretamente Arvicola terrestris cantiana.

NoROESTE.

Waverley Wood (Rose 1987)

Se presentó como la evidencia más antigua del poblamiento de las islas británicas. En este sitio el problema no es determinar el carácter antrópico de la industria. Es claramente humana. Se trata de tres bifaces bastante simétricos y una serie de lascas halladas en la base de un depósito de gravas. El problema es aceptar la fecha de unos 600.000 años que se atribuye al sitio. Tanto los datos litoestratigráficos como faunísticos son consistentes con una cronología situada entre los estadios 13 o comienzos del 12, en torno a los 500.000 años.

Boxgrove (Roberts 1990, Gamble 1993)

Es el primer sitio con un contexto en posición primaria en esta zona. Se le considera contemporáneo a sitios como Miesenheim, en el estadio 13 , en torno a esa fecha del 0,5 millones de años.

\section{Macizo Central Francés}

En esta región montañosa del centro de Francia entre el río Ródano y regiones como la Champagne, Poitou, Perigord o los Pirineos, se encuentran faunas del Pleistoceno antiguo con buerios contextos estratigráficos. En algunos de estos sitios se han recogido conjuntos líticos. Cuando el contexto estratigráfico parece bien definido el problema pasa 
a ser el determinar el carácter humano o no humano de tales conjuntos líticos.

Chilhac III (Chavaillon 1991, Gutch \& Chavaillon 1985)

Chavaillon describe 46 piezas de indudable manufactura humana, fabricados sobre basalto, rocas metamórficas y cuarzo. No obstante, muestra cierta incertidumbre al inclinarse por su edad. Los artefactos fueron hallados en unos niveles de gravas y arenas (B3, $G$ y K) que alternan con niveles de arcillas y arenas que contienen únicamente fauna. La fauna encontrada en los niveles de gravas y algunos de los artefactos líticos están abrasados mientras que la fauna encontrada en los niveles de arcillas no. El sitio tiene una datación de 1,9 millones de años, según los datos paleontológicos y los que se obtienen de una colada de lava. De ser cierta esta interpretación, pasaria a ser el sitio más antiguo de Europa. Los niveles presentan un fuerte grado de inclinación y habría que tener en cuenta posibles fenómenos de solifluxión. Texier (1985) describe la formación del sitio: niveles de grano fino (arcillas y cenizas volcánicas) fueron depositados originalmente en el fondo de un antiguo lago. Posteriormente fueron erosionados y transportados por gravitación para ser mezclados con aportes coluviales y finalmente ser redepositados. Si esta interpretación estratigráfica es correcta, los artefactos podrían no ser contemporáneos a la fauna encontrada en el sitio.

Soleilhac (Bonifay 1987)

No existe ninguna publicación en detalle de este sitio. Se trata de una serie de pebbles tools en cuarzo que podrían resultar más de una serie de fracturas naturales que de un trabajo de talla o lascado. Es un conjunto muy dudoso si se compara con fragmentos de basalto no modificados y se podría hablar de una selección de objetos por una morfología "primitiva".

Le Vallonet (Lumley 1988, Lumley et al. 1988)

Este sitio contiene sedimentos datados en torno al millón de años por estudios bioestratigráficos, paleomagnéticos y dataciones absolutas (ESR). Descrito como rico en fauna y con muy escasa industria, ésta se sitúa en la Unidad III (B1, B2, C) formada por arenas y margas con una gran cantidad de cantos y guijarros angulosos. Estos depósitos se colaron en la 
cueva a través de una chimenea y una serie de fisuras dando lugar a la Unidad III. Después sufrió una fuerte exposición a fenómenos geoquímicos con toda clase de alteraciones sobre cantos y guijarros: "Les cailloux et les galets de ces niveaux sont souvent craquelés avec déplacements de fragments» (Lumley 1988, pág. 416). La industria se compone de unas setenta piezas y es descrita como representativa de una tecnología muy primitiva. La materia prima es una caliza extraída de un conglomerado de edad Miocénica. Se encuentran pebbles, pebbles tools, choppers, chopping tools, lascas, sin ningún tipo de estandarización y de una calidad muy mediocre. Están muy deformadas, descarbonatadas, fragmentadas y craqueladas entre otras alteraciones. Para Roebroeks y Van Kolfschoten se ha producido una selección de piezas de un depósito natural por su aspecto "primitivo" sin que éstas hayan sido modeladas por el ser humano.

\section{Peninsula lbérica}

\section{Venta Micena (Gibert 1985)}

Este controvertido sitio al que ya nos hemos referido, se localiza en la región de Orce, en la denominada formación de Baza. Por la composición de la fauna se proponen fechas entre 1,6 y 0,9 millones de años. Pese a que los excavadores registran la existencia de un fósil humano, la falta de instrumentos líticos coloca el sitio bajo un gran interrogante. Hasta el momento lo prudente es ser escépticos en relación a la presencia humana en este yacimiento.

Cúllar-Baza (Ruiz Bustos et al 1986)

El sitio se encuentra a unos veinte kilómetros al suroeste de la localidad del mismo nombre. Se describen seis lascas y dos choppers asociados a una fauna de Pleistoceno Medio. Serian los artefactos más antiguos de la zona de la depresión Guadix-Baza. Sin embargo, existen problemas en el grado de asociación con la fauna, ya que el conjunto faunístico pudo acumularse debido a la acción de depredadores y otra serie de agentes no humanos.

Atapuerca (Carbonell y Rodríguez 1994)

La Sierra de Atapuerca se encuentra a $14 \mathrm{~km}$ al este de Burgos. Dos son los niveles que nos interesa revisar brevemente aquí. Ambos se encuen- 
tran en la estratigrafia del relleno conocido como "Gran Dolina", con unos $18 \mathrm{~m}$ de potencia. TD4 es un nivel con una abundante fauna y una escasa industria. Podría situarse entre los estadios de la curva isotópica 18 y 17 , con fechas entre 726.000 y 659.000 años. No obstante, el carácter humano de los cinco artefactos en cuarcita es dudoso. TD6 es un paquete de unos doscientos centímetros, de arenas con una elevada fracción arcillosa y abundantes clastos calcáreos. Presenta un aspecto caótico en su parte proximal y una clara estratigrafía en los últimos $50 \mathrm{~cm}$. Existe una abundante fauna e industria lítica. Se compone de una treintena de piezas. Aproximadamente el $65 \%$ de la materia es sílex, que puede conseguirse a poca distancia del sitio, en un radio de unos $3 \mathrm{~km}$. Aparecen algunos bifaces. La fecha para este nivel se sitúa tentativamente en unos 0,5 millones de años, en buena consonancia con el resto del registro europeo.

Península itálica

Existen muchos hallazgos en superficie a los que se atribuye una gran antigüedad por las morfologias «primitivas» de las piezas líticas. El problema una vez más es identificar o correlacionar a falta de contextos estratigráficos, tipología y cronología.

Monte Poggiolo (Gagnepain et al. 1992)

El sitio presenta dataciones absolutas entre 1,3 y 0,73 millones de años. Sin embargo, problemas de su contexto estratigráfico impiden que pueda hablarse con firmeza de una evidencia de presencia humana en la zona para esas fechas.

Isernia La Pineta (Peretto et al. 1991)

Pese a algunas dataciones radiométricas (Coltorti et al. 1982), los datos paleontológicos demuestran que la fecha no puede remontarse más allá de 0,7 millones de años como mucho. La presencia de Arvicola terretris cantiana sólo se explicaría en fechas anteriores como una población primitiva. Esta solución no parece convincente y es problemática pues implicaria la existencia de una serie de barreras naturales que no parecen haberse dado durante todo el Pleistoceno a juzgar por la composición de las faunas de los diversos sitios europeos. Una alternativa acorde con ese límite cronológico en los 700.000 años es que estemos ante un cambio gradual de las faunas villafranquienses a galerienses. 
Las primeras evidencias con contextos claramente primarios se encuentran en yacimientos como Fontana Ranuccio, Visogliano o Venosa, todos ellos con fecha asimilables a las obtenidas en sitios como Boxgrove o Karlich $\mathrm{G}$.

Pese a que han aumentado las técnicas y posibilidades de las dataciones absolutas, el marco de fechas absolutas para el Pleistoceno Medio es escaso y presenta dificultades a la hora de establecer correlaciones entre diferentes regiones. Los márgenes de error son grandes y dificultan la posibilidad de establecer correlaciones sincrónicas entre sitios de diferentes regiones, que nos ayuden a realizar inferencias paleoecológicas y reconstruir patrones de comportamiento de estos grupos humanos ${ }^{3}$.

Todos los sitios que hemos revisado hasta aquí tienen una problemática y unas carencias comunes que les restan solidez y les colocan en el escepticismo o en el descrédito. Esas carencias provienen esencialmente de la falta de contextos estratigráficos y de la dificultad para identificar el carácter humano de los artefactos líticos. Se trata de sitios que presentan colecciones recogidas en superficie, sin contexto estratigráfico, en posiciones derivadas y faltas de homogeneidad.

Nada parece indicar a la vista de lo expuesto que los humanos fuesen los autores de esas colecciones. En muchos casos parece haberse dado una selección de piezas, extraídas de un contexto de cantos y guijarros, en base a una morfologia que recuerda una tipología producida por una tecnología "muy primitiva". Sin embargo, es sumamente complejo establecer una correlación entre tipología y cronología y mucho más sin contextos estratigráficos bien determinados. A esto se suma la práctica inexistencia de gestos técnicos discernibles en la fabricación de tales artefactos como la presencia de bulbos, negativos $u$ ondas de percusión. Otro factor más es la falta de homogeneidad que presentan las piezas de una misma colección en cuestiones como su estado de conservación entre otras.

Si observamos pues estas conclusiones y las añadimos a las que obteníamos de la revisión del registro fósil humano, parece prudente con-

\footnotetext{
3 En la Peninsula lbérica esta situación es especialmente acusada con dataciones absolutas escasas y una falta de conocimiento de los grandes eventos climáticos del Pleistoceno: "The Alpine sequence is inapplicable, since the interior mountains preserve no clear evidence of pre-Würmian glaciations. (...) In this situation, correlation with deep-sea cores or the paleomagnetic record would be insecurely based and any such proposals are extremely tentative." (Santonja \& Villa, 1990).
} 
cluir que sólo podemos hablar en nuestro estado actual de conocimientos de una primera presencia efectiva de homínidos en Europa a partir del Pleistoceno Medio. Los apoyos para una cronología baja pueden resumirse en los siguientes puntos (fig. 3):

a) Inexistencia de pruebas sólidas anteriores al Pleistoceno Medio. Se trata de escasos sitios y con contextos en posiciones derivadas frente al aumento de los sitios en cantidad y calidad, con buenos contextos en posición primaria, a partir del Pleistoceno Medio en toda Europa.

b) Problemas en la cronología de tales evidencias debidos a la propia naturaleza de los hallazgos desprovistos de contextos estratigráficos.

c) Dificultad para discernir o identificar la participación humana en la fabricación de los artefactos líticos. Imposibilidad de interpretar gestos técnicos ante la ausencia de posibles bulbos, negativos $u$ ondas de percusión.

d) La ausencia de restos fósiles humanos con anterioridad al Pleistoceno Medio.

Sitios anteriores a 0.5 millones

de años.

- series pequeñas de piezas aisladas y seleccionadas de un fondo de

guijarros fracturados naturalmente.

- contexto estratigráfico inexistente o derivado: en el mejor de los casos.

- material lítico muy controvertido y con dudas sobre su carácter antrópico.

- inexistencia de restos fósiles humanos.
Sitios posteriores a 0.5 millones de años.

- amplias colecciones obtenidas de "talleres líticos" con remontaje de piezas.

- estratigrafias bien determinadas y sitios en contexto primario

- descripción de claras industrias achelenses y no achelenses.

- restos fósiles humanos.

Fig. 3. Caracteristicas del registro paleolitico anterior y posterior al Pleistoceno Medio. (Extraido de Roebroeks \& Van Kolfschoten 1994). 
Con estos datos optamos por una cronología baja para el poblamiento de Europa que toma como fecha convencional 0,5 millones de años. Con independencia de ocupaciones intermitentes anteriores que pudieran demostrar en un futuro yacimientos como Chilhac III, resulta evidente que el poblamiento efectivo sólo puede remontarse al Pleistoceno Medio. Poblamiento efectivo que por otra parte debe ser a nuestro juicio el verdadero objeto de estudio para los investigadores.

¿Qué conclusiones surgen de forma inmediata, si aceptamos una cronología baja para el poblamiento de Europa, a la vista de los datos que aporta el registro arqueológico?

a) Fue el continente ocupado más tardiamente con independencia del continente americano. Habría que preguntarse por qué los homínidos salidos de Africa se detuvieron en las puertas de Europa y esperaron cerca de medio millón de años antes de entrar.

b) Una vez que los sitios demuestran una ocupación humana de Europa indiscutible, podemos afirmar observando la distribución de los mismos en el mapa de Europa que ésta se produce en todo el suelo europeo de forma sincrónica (fig. 4).

\section{LA CUESTIÓN DEL MEDIO}

Estas dos conclusiones son sumamente interesantes ya que plantean una serie de interrogantes muy significativos en relación a las pautas de comportamiento que debieron regir a estos grupos. ¿Qué implica el hecho de que la ocupación fuese tardía y de que una vez que se produjo, ésta fuera prácticamente sincrónica en toda Europa, a la hora de enfrentarse a la configuración de un patrón o modelo que reconstruya el comportamiento de los primeros Homo erectus llegados a Europa?

Sin duda debemos contemplar la salida de los homínidos de Africa dentro de un fenómeno biogeográfico amplio de irradiación de varias especies de mamíferos hacia Asia y Europa en torno al millón de años, ¿por qué los homínidos tardaron tanto en ocupar nuestro continente? Yacimientos como Dmanisi (Cáucaso), Ubeidiya (Oriente Próximo) con fechas de 1,25 millones de años o las industrias con un achelense muy antiguo en contextos de hace un millón de años para la zona del Magreb africano; prueban que los homínidos se detuvieron ante las puertas de Europa y esperaron allí medio millón de años antes de entrar. Los investigadores se preguntan ahora ¿a qué se debió esa larga espera?, ¿podemos pensar que el medio ambiente debió de jugar un papel importante en tal lapso de tiempo actuando como una barrera a la expansión homi- 


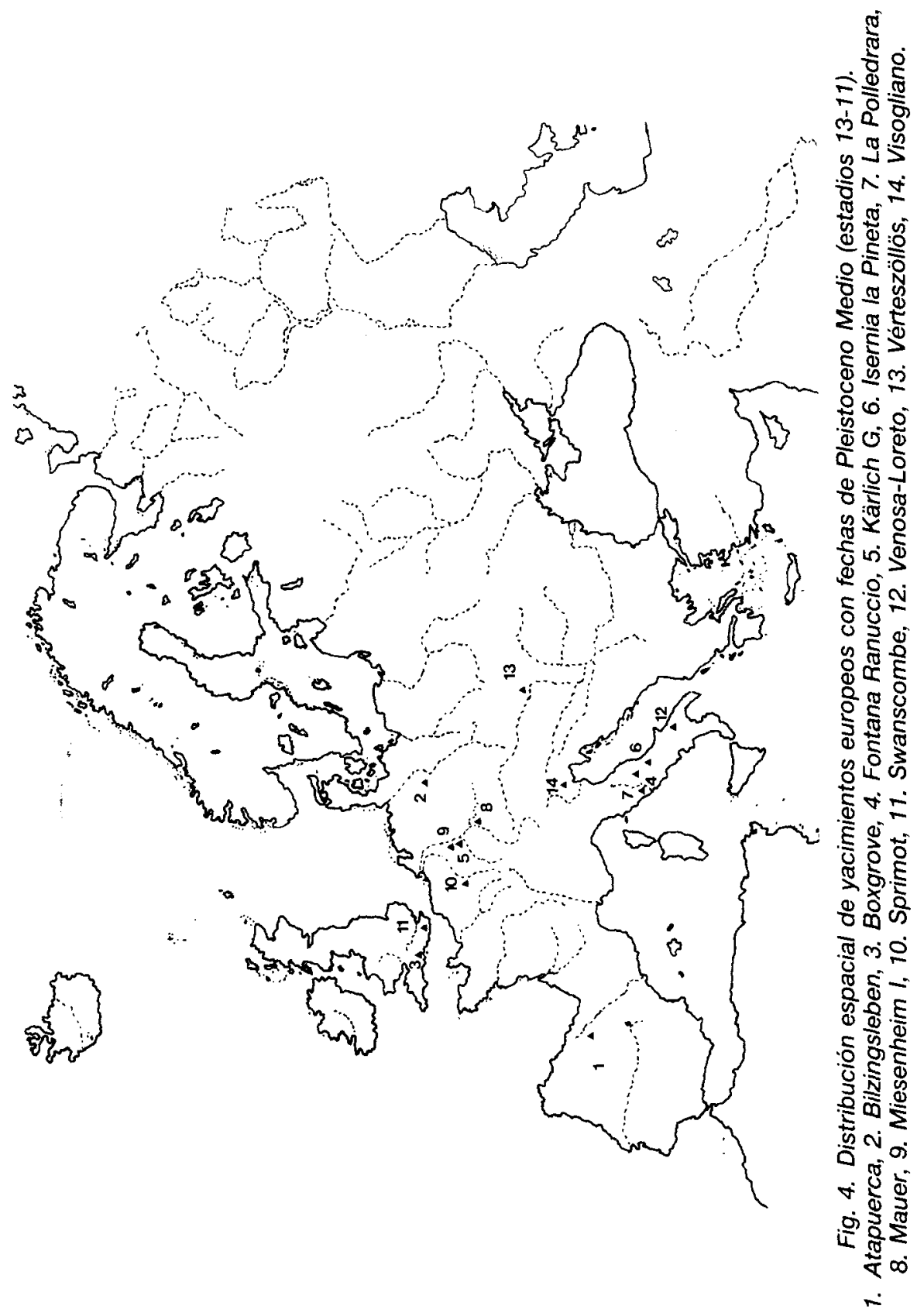


nida? Las condiciones ecológicas que se daban en Europa hace un millón de años pudieron ser en efecto una barrera infranqueable para su poblamiento por humanos. ¿Poseian unas estructuras sociales, unas estrategias de subsistencia y un equipamiento tecnológico capaces de enfrentarles con éxito a un nuevo marco ecológico diferente de aquel del que provenian? La presencia humana en zonas como Marruecos o el Cáucaso hace un millón de años viene a demostrar un asentamiento en lugares preferentemente de medios abiertos y entornos más similares a las sabanas africanas que los hábitats principalmente boscosos de Europa. Esto ha llevado a algunos autores (Gamble) a plantear la existencia de una barrera ecológica que los homínidos sólo fueron capaces de superar hace medio millón de años. A esa barrera se la ha denominado «el telón de bosques" (the wooden curtain).

Sea como fuere, es un hecho que en torno al medio millón de años se produjo la colonización de Europa de una forma efectiva, lo que demuestra que poseían las estructuras biológicas, sociales y económicas necesarias para sobrevivir en todos los medios y hábitats europeos. Si esos hábitats fueron en un primer momento una dificultad insuperable, lo cierto es que medio millón de años después el medio ambiente no impuso prácticamente límites a la ocupación. Toda una serie de yacimientos europeos con una gran variedad de latitudes y cuyas dataciones giran en torno a esa fecha convencional de 500.000 años vienen a demostrar esta afirmación (fig. 5).

Ante estas evidencias lo que debemos preguntarnos no es si existió una llegada de grupos humanos a Europa con anterioridad a 0,5 millones de años, que en todo caso estaría reflejando llegadas ocasionales y asentamientos intermitentes. Por el contrario, debemos interesarnos por los factores que permitieron un poblamiento permanente y efectivo de tales tierras. Qué factores, si es que los hubo, ecológicos, climáticos, geográficos, económicos y sociales posibilitaron una primera colonización efectiva que se propagó como una ola por todo el continente con un escaso margen de tiempo, de forma prácticamente sincrónica, desde las latitudes más mediterráneas a las más cercanas a los fríos de las grandes masas de hielo.

Los investigadores se preguntan si existió entonces un límite norte para estos pobladores. El vacio que presenta la región escandinava en yacimientos de paleolítico inferior podría entenderse como una respuesta afirmativa. Sin embargo, los mismos investigadores se preguntan si ese vacío de yacimientos es real o se debe a una falta de condiciones que posibiliten su conservación debido a la acción de los glaciares durante todo el Pleistoceno. Sitios como Pontnewydd (Gales) demuestran que se poblaron zonas con latitudes muy altas. También es cierto que existen vacios de sitios de paleolitico inferior en zonas mucho más al sur, 


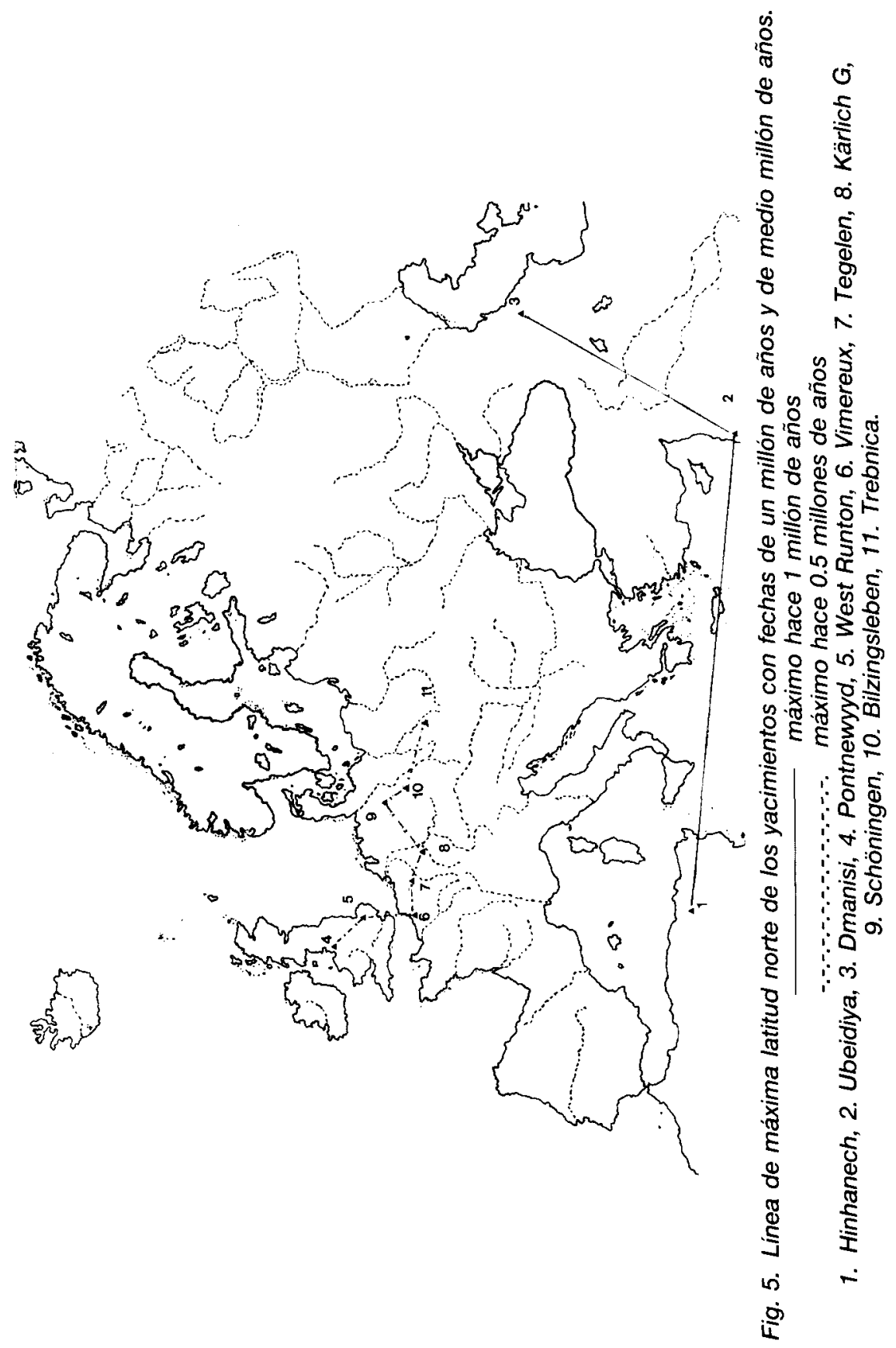


como por ejemplo el valle del Ebro, en la península Ibérica. Esto viene a evidenciar la necesidad de potenciar los estudios paleoecológicos que puedan darnos la clave a tales vacíos.

Es aquí donde cobran pleno sentido las propuestas de estudios regionales de investigadores como Gamble (1986). Desde una perspectiva que podriamos denominar como "Ecología humana", los modelos regionales imponen esa necesidad de los estudios paleoclimáticos. Estudiar ese fenómeno demográfico que fue el poblamiento del continente europeo durante el Pleistoceno exige un perfecto conocimiento del medio en el que esos pobladores se movieron tanto a un nivel regional como a un nivel local: la geografía, el clima, etc... Sólo así podemos comprender el papel que pudieron jugar en ese fenómeno factores como la tecnología, los potenciales recursos, la competencia con otras especies o las estrategias de subsistencia entre otros muchos.

¿Cuáles pudieron ser las barreras superadas en torno a los 500.000 años?

a) Ecológicas. Claramente podemos incluir aqui factores como el clima (temperaturas, estaciones, horas diarias de luz, etc...), las características de los hábitats (recursos potenciales, competición con otras especies...).

b) Económicas. Sin duda muy condicionadas por el apartado anterior, las estrategias empleadas para obtener los recursos alimenticios y de otro tipo (vestimenta, energía, infraestructura...), caza, carroñeo. Las capacidades tecnológicas, la explotación de recursos, la localización de asentamientos, etc...

c) Sociales. Principalmente la emergencia y dispersión de las redes de emparejamiento o la interacción que tuvo que darse entre la organización social de estos grupos y sus estrategias de subsistencia.

d) Biológicas. La posibilidad de un mayor desarrollo del cerebro y del sistema nervioso o de unas capacidades cognitivas mayores para comunicarse por ejemplo mediante un lenguaje hablado, etc...

Todos los especialistas se muestran de acuerdo en la necesidad de profundizar en los estudios paleoecológicos para encontrar respuestas a estos planteamientos. Esos estudios deben realizarse tanto a nivel regional como a nivel local. A nivel regional es interesante observar las variaciones diacrónicas y también las sincrónicas entre diferentes medios regionales para intentar establecer correlaciones. Podemos encontrar de este modo posibles respuestas a los vacíos de registro arqueológico en algunas zonas o comparar las amplitudes y efectos sobre el medio de los ciclos glaciares-interglaciares en regiones muy distantes, y poder inte- 
rrelacionar toda esa información con las adaptaciones que hicieron efectivo el poblamiento de Europa. Estas tuvieron que ser soluciones a largo plazo y en modo alguno oportunistas. A nivel local el interés se centra en conocer adaptaciones concretas a medios concretos. Los sitios de Paleolítico Inferior parecen tener la constante de ser sitios al aire libre, en la mayoría de los casos los medios de deposición se corresponden con medios riparos, lacustres o palustres. Esto conlleva una serie de interrogantes de los que trataremos en el apartado siguiente.

Es común en tales sitios la asociación de restos de fauna y restos líticos, aunque la interacción humana con estos restos faunísticos es muchas veces dificil de demostrar.

\section{LA CUESTIÓN DE LA SUBSISTENCIA}

Al hilo de lo anterior la primera afirmación clara es que resulta muy complicado conseguir una transformación importante de los datos medioambientales en datos económicos que nos informen de la subsistencia de estos grupos. El registro arqueológico del paleolítico inferior es en general un registro muy fragmentado y sesgado. Los datos que nos proporcionan son escasos, complejos y precarios con raras excepciones (Boxgrove, Aridos...). Ello se debe a la existencia de una gran cantidad de elementos que vienen a actuar sobre su conservación. Lo que resulta obvio es que si queremos acercarnos a los procesos de tipo económico, las claves tenemos que encontrarlas en este registro. La mejor manera de paliar las deficiencias que presenta es estudiando en detalle las características de los sitios y fundamentalmente su proceso de formación. Como hemos comentado ya, se trata de sitios al aire libre y generalmente en medios fluviales o lacustres. ¿A qué responde esto?, ¿supone un sesgo en el registro debido a problemas de conservación? "La probabilidad de que un yacimiento paleolitico haya perdurado está totalmente condicionada por la presencia de depósitos capaces de conservar registro arqueológico, y por tanto hay un sesgo que favorece claramente al medio fluvial, e incluso más aún -aunque ocupe extensiones más reducidas- al lacustre. No existen prácticamente buenos yacimientos fuera de estos ámbitos a causa de las reducidas posibilidades de conservación en condiciones apropiadas." (Santonja 1992). Estos medios presentan yacimientos ligados casi siempre a un tipo de actividad muy concreta, los denominados "butchering sites" o lugares de despedazado de animales. Cabe la posibilidad de que con la pérdida de yacimientos depositados en otros tipos de medio que favorecen poco su conservación hayamos perdido información de otros muchos tipos de 
actividades. Los «butchering sites» son sitios que presentan un alto grado de resolución e integridad (Gamble 1990): «Un solo agente es el responsable de la acumulación de material. Desempeña una única actividad. El sitio generalmente no se reutiliza." Son los más propicios para el hallazgo de suelos de ocupación proporcionando una importante información económica: "los materiales depositados reflejan de una manera más precisa las actividades que se llevan a cabo en ese lugar y en relación con el entorno inmediato." En efecto, nos dan la posibilidad de reconstruir actividades muy concretas, despedazado de grandes animales para la obtención de alimentos u otros recursos básicos, con independencia de si éstos fueron obtenidos mediante estrategias de caza o de carroñeo. Si pensamos en una estrategia de carroñeo, nos hablan del desplazamiento de un grupo humano a un lugar donde se encuentra carroña, si pensamos en una estrategia de caza tenemos que admitir un alto grado de organización y planificación en las estrategias económicas de estos grupos. Se trata de ocupaciones breves a lo sumo un par de semanas $y$ donde se utiliza un utillaje adaptado a unas necesidades muy precisas. El hecho de que se trate de yacimientos generalmente depositados en fondos de valles y ambientes fluviales como llanuras de inundación plantea interrogantes de gran interés:

a) ¿La estrecha relación que se da entre estos medios y este tipo de yacimientos se debe a problemas de conservación y su posible existencia en otros medios de deposición se nos escapa?

b) Si admitimos esa estrecha relación como fruto de factores ecológicos y económicos y no de cuestiones ligadas a la conservación, podemos preguntarnos si se dio la existencia de recursos previsibles, si los erectus eran conscientes de ello y por último si fueron capaces de explotarlos.

Sin duda son sitios privilegiados por la información que desprenden acerca de las actividades económicas. La investigación futura debe dirigir sus esfuerzos en dos direcciones paralelas. Por un lado, seguir trabajando en la documentación y elaboración de los datos que nos proporcionan los suelos de ocupación y por otro profundizar en estudios de carácter tafonómico, el proceso de formación de los sitios donde se documenta la existencia de más de una actividad. La tafonomía nos puede acercar a comprender qué tipo de información podemos extraer de cada yacimiento, qué preguntas están capacitados para respondernos.

\section{LA CUESTIÓN DE LAS INDUSTRIAS}

Dejando a un lado el tema que ya hemos comentado del posible carácter antrópico o geológico de las evidencias líticas, lo que nos inte- 
resa destacar aqui es que todo parece sugerir que los útiles de piedra del Paleolítico Inferior carecen de un valor cronológico absoluto y sólo sirven como marcadores de una presencia humana en puntos especificos en el tiempo.

Lo que sí es interesante es analizar la variación de las industrias del Paleolítico Inferior desde una doble vertiente, la temporal y la espacial.

\section{La variación temporal}

En un principio resulta muy tentativo correlacionar un posible desarrollo tecnológico con avances o cambios en las estrategias de subsistencia, así como con la organización social. El primer problema que plantea un análisis de la variación temporal es cuestionarnos si las tecnologías y los hábitos o tradiciones culturales son lo suficientemente estables o no en el tiempo como para posibilitar tal análisis, máxime cuando hemos de admitir que las tendencias en el tiempo no son siempre unilineales ni irreversibles. Partiendo de estas premisas lo que se puede concluir es que con el tiempo se va incrementando el refinamiento y el cuidado en la elaboración de las piezas y conceptos como la simetria se van haciendo patentes. Comienza a detectarse poco a poco una cierta estandarización en la fabricación de los útiles (la técnica levallois), un mayor peso en la utilización de los percutores blandos, la búsqueda de formas más definidas y la creación de un utillaje más variado en lascas. La cuestión a solucionar es si todas estas tendencias conllevan o son un reflejo de cambios en las estrategias de subsistencia y en la organización social.

\section{Variación espacial}

La problemática de la existencia de industrias achelenses y no achelenses a la que se une la presencia de industrias con un utillaje con tendencia al microlitismo en yacimientos como Bilzingsleben o Isernia la Pineta, parece descartar las soluciones de carácter cronológico. No está claro que se sucedan unas a otras en el tiempo o que se excluyan mutuamente. Las respuestas pueden encontrarse en cuestiones vinculadas a necesidades adaptativas o a imposiciones de la materia prima.

Convendria tener en cuenta una variedad de factores:

a) La facilidad/dificultad para obtener materia prima y las imposiciones e influencias que ejerce sobre cuestiones como la tecnología o la morfología de las industrias. 
b) La naturaleza de la ocupación donde esos conjuntos líticos son registrados.

c) El posible peso que puedan ejercer las tradiciones culturales.

Pueden intervenir otras muchas razones, sin embargo, parece que 10 más prudente en estos momentos es conceder un mayor peso a las condiciones de la materia prima: "Tal influencia de la materia prima en el aspecto y la composición de las industrias es un factor que conviene sopesar en primer lugar, antes de buscar explicaciones de indole étnica o cultural -circunstancias que ignoramos totalmente si y cómo pudieron haberse manifestado en "Homo erectus" - a las diferencias que aquellas ofrecen. Incluso esta posibilidad también deberia contrastarse antes de ver en las diferentes "tradiciones culturales" mecanismos de adaptación al medio ambiente..." (Santonja 1992).

En una escala amplia se constata claramente que pese a las diferencias existe un alto grado de universalidad y homogeneidad en las industrias del paleolítico inferior. No existe pues una correlación clara entre la diversidad de medios y la que presentan las industrias líticas, esa diversidad de medios no se refleja. Cierto es que como ya hemos dicho, resulta sumamente complejo realizar comparaciones a nivel regional porque faltan dataciones absolutas que permitan establecer sincronias. No obstante, esa homogeneidad en las industrias puede que esté reflejando una escasa capacidad de adaptación a diferentes medios, como ha señalado Villa (1991).

\section{CONCLUSIONES}

1. A la vista de los datos que nos aportan el registro fósil humano y el registro arqueológico de los sitios europeos, parece evidente que sólo puede hablarse de un poblamiento efectivo del continente en el Pleistoceno Medio en torno a la fecha convencional de 0,5 millones de años. Una vez que se produce la ocupación del continente europeo, ésta se realiza en la práctica totalidad de los territorios y con un escaso margen de tiempo. En tal fecha parecen romperse una serie de barreras ecológicas, económicas, sociales y biológicas que hacen posible esa ola sincrónica de asentamiento.

2. Si la aceptación de una cronología alta habria hecho necesaria la hipótesis de una adaptación gradual de los erectus a un amplio rango de hábitats europeos, donde en un lapso de un millón de años se habría producido ese proceso gradual de adaptación desde las primeras industrias de cantos a la aparición del achelense; por el contrario, la aceptación de 
una cronología baja obliga a contemplar ese proceso de adaptación como más rápido, no atribuyendo ningún valor cronológico a las industrias sin bifaces o con bifaces y destacando la existencia desde un principio de industrias achelenses en yacimientos como Boxgrove, Atapuerca TD6 o los conjuntos más antiguos de las terrazas del Somme en Abbeville (Francia).

3. ${ }^{\circ}$ El éxito del poblamiento de Europa se debió a la combinación de unas estrategias de subsistencia a largo plazo adaptadas a los ritmos ambientales del Pleistoceno. Esas estrategias estuvieron determinadas por el medio y condicionadas por la organización social (fig. 6). Y esto es así porque es el medio el que determina qué recursos podian explotar y cuándo podían hacerlo; y porque es la organización social la que impone cómo se pueden explotar esos recursos, pese a que es indudablemente el modo de producción el que determina el carácter de la formación social. $Y$ es evidente desde esta perspectiva que para que esos grupos desarrollasen unas estrategias capaces de imponerse al mayor o menor grado de determinismo medioambiental, las soluciones de carácter social debieron tener prioridad a las innovaciones tecnológicas.

4. ${ }^{\circ}$ Todo parece indicar a partir del análisis de las materias primas y de los territorios de captación de la misma que la vida de estos grupos fue muy local y que en general se dio un uso muy episódico de los sitios que nos han llegado.

5. La existencia y necesidad de adaptarse a medios tan diferentes y la constatación de las evidencias líticas pueden indicar:

a) Que se dieron estrategias adaptativas diferentes. Si fue así, ¿cuáles fueron las semejanzas y diferencias y en qué escala?

b) Que como parece indicar la homogeneidad de las industrias del paleolítico inferior, las estrategias fueron similares y lo suficientemente flexibles como para resultar exitosas en medios tan diferentes, o bien demuestran una escasa capacidad de adaptación a medios ecológicos diferentes.

Lo que sin duda tuvo que darse es un cierto grado de planificación y organización consciente en las actividades económicas que se demuestra en el éxito de una adaptación a largo plazo. La posible existencia de estrategias de carroñeo vinculadas a una explotación planificada de grandes mamíferos en entornos previsibles como podrian estar reflejando los «butchering sites" podria entenderse como un paso intermedio a estrategias que exigen de un mayor grado de organización como la caza. Esto nos llevaría a preguntarnos si se produjo una evolución de las estrategias a lo largo del Pleistoceno hacia un mayor grado de organización y planificación con los cambios que ello supondría a nivel de la sociedad; o si existió desde el principio una amplia variedad y flexibilidad de comportamientos 
vinculados con la subsistencia. Habria que preguntarse si el continente europeo poseía la suficiente diversidad de marcos ambientales que dieran lugar a esa amplia variedad de comportamientos, como consecuencia de la presión selectiva que opera en las estrategias de subsistencia (Gamble 1986). Para encontrar respuestas a estas preguntas resulta obvio que:

6..$^{\circ}$ Se necesita un mayor cuadro de dataciones absolutas y de reconstrucciones paleoclimáticas a nivel regional y local que nos permitan establecer sincronías y correlacionar los datos de las regiones más distantes en el intento por reconstruir o al menos representar algunos componentes del comportamiento humano.

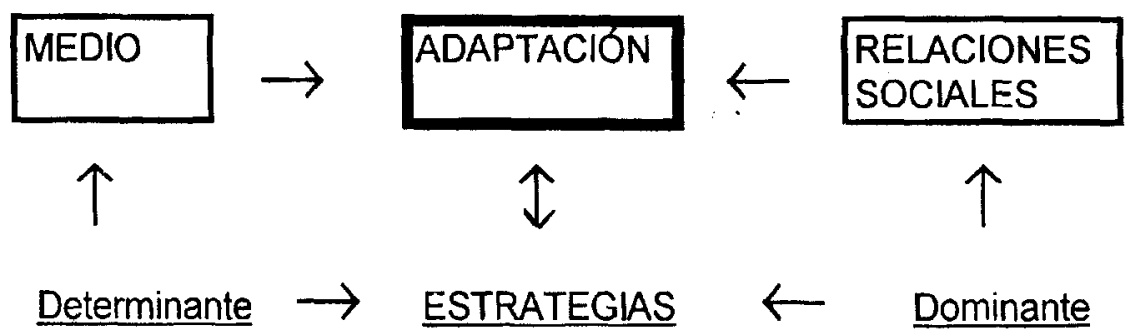

Fig. 6. Relaciones existentes entre medio, organización social y estrategias de subsistencia.

"El medio ambiente puede clasificarse en primera instancia como determinante, estipula de qué se dispone para su explotación y cómo, dentro de unos márgenes muy amplios, se tendrá que conseguir mediante la manipulación del tamaño del grupo, el número de desplazamientos, la dirección y ubicación de la residencia. Por encima del medio hay otro nivel, mientras que las relaciones ecológicas pueden ser determinantes, las relaciones sociales deben considerarse como dominantes en última instancia porque especifican cómo se ha de explotar el entorno.

Sistema social, modos y relaciones de producción establecen el modelo de explotación.» (GAMBLE 1990)

Las últimas dataciones propuestas para los hallazgos de restos de homínidos en Gran Do!ina TD6 en Atapuerca (Burgos) en torno a los 800.000 años, junto a las industrias recogidas en los yacimientos; Barranco del León y Fuentenueva 3a en la región de Orce (Granada) atribuidas, aunque sin fechas bien determinadas aún, a un momento del Pleistoceno Inferior, han abierto interrogantes muy sugerentes sobre la cuestión del poblamiento de Europa y la existencia para el mismo de una cronologia alta o baja. Tales planteamientos fueron puestos sobre la mesa de debate del Congreso Internacional de Paleontología Humana celebrado en Orce durante los días 4 a 7 de septiembre de 1995 , coincidiendo todos los participantes en el enorme interés que estos nuevos datos vienen a aportar. Queda claro que desde ahora se abre una perspectiva investigadora en esta cuestión con un futuro muy interesante y desde luego nunca falto de controversias e interés cientifico. 


\section{BIBLIOGRAFIA}

ACKERMAN, S. (1989): "European prehistory gets even older", Science, 246: 28-30.

AguiRre, E. (1991): "Les premiers peuplements humains de la Péninsule lbérique", In BONIFAY \& VANDERMEERSCH (1991): 143-150.

BONIFAY, E. (1987): Soleihac 1987. Rapport de fouilles. Marseille: Laboratoire de Géologie du Quaternaire.

Bonifay, E., y Vandermeersch, B. (1991): Les Premiers Européens. Paris: Editions du C.T.H.S.

Carbonell, E., y Rodríguez, X.P. (1994): "Earley Middle Pleistocene deposits and artefacts in the Gran Dolina site (TD4) of the "Sierra de Atapuerca" (Burgos, Spain)", Journal of Human Evolution, 26: 291-311.

CLARK, J.D. (1958): "The natural fracture of pebbles from the Batoka Gorge, Nothern Rhodesia, and its bearing on the Kafuan industries of Africa", Proceedings of the Prehistoric Society, 24: 64-77.

Coltorti, M.; Cremaschi, M.; Delitala, M.C., et al. (1981): "Reversed magnetic polarity at an early Lower Palaeolithic site in Central Italy", Nature, 300: 173-176.

Cook, J.; Stringer, C.B.; Currant, A.P.; Schwarcz, H.P., y Wintle, A.G. (1982): “A review of the chronology of the European Middle Pleistocene hominid record", Yearbook of Physical Anthropology, 25: 19-65.

Chavalloon, J. (1991): "Les ensembles lithiques de Chilhac III (Haute Loire): typologie, situation stratigraphique et analyse critique et comparative", In Bonifay \& Vandermeerch (1991): 81-91.

Delson, E. (1989): “Oldest Eurasian stone tools", Nature, 340: 96.

DzAPARIDZE, V.; BosinkI, G.; BugIANISVILI, T., et al. (1989): “Der altpaläolithische Fundplatz Dmanisi in Georgien (Kaukasus)", Jahrbuch des Römisch=Germanischen Zentralmuseums Mainz, 67-116.

FRIDRICH, J. (1989): Prezletice: A lower Palaeolithic site in central Bohemia (Excavations 1969-1985). Praga: Museum National Pragae.

- (1991): "The oldest Palaeolithic stone industry from the Beroun highway complex", Antropozoikum 20: 111-128.

GAGNEPAIN, J.; HEDLEY, I.; BAHAIN, J.-J. y WAGNER, J.-J. (1992): “Etude magnétostratigraphique du site de Ca'Belvedere di Monte Poggiolo (Forli, Italie), et de son contexte stratigraphique. Premiers résultats", In PeRETTO (1992): 319-335.

GamBLE, C.S. (1986): The Palaeolithic settlement of Europe. Cambridge University Press. Cambridge. Hay traducción en castellano; El poblamiento Paleolítico de Europa (1990). Ed, Crítica, Barcelona.

- (1993): Timewalkers. The Prehistory of Global Colonization. Stroud (Gloucestershire): Alan Sutton.

GiBerT, J. (1985): “Venta Micena: un dels jaciments humans més antics d'Europa”, Tribuna d'Arqueologia 1983-1984. Dir. Gen. Patrimoni Artistic, Servei d'Arqueología, Barcelona, págs. 53-58.

Guth, C. y Chavaillon, J. (1985): “Découverte, en 1984 de nouveaux outils paléolothiques à Chilhac III. (Haute Loire)", Bulletin de la Société Préhistorique Française, 82: 56-64.

Guthrie, D. (1984): “The Galerian dispersal event and the origin of the modern megafauna, its implications for Earley Man in the Northern Hemisphere", The Quarterly Review of Archaeology, 5(2): 15-16.

KozLOWSKI, J.K. (1992): “Les premiers habitants de l'Europe centrale et orientale", In PERETTO (1992): 69-91.

LUMLEY, H. DE (1988): "La stratigraphie du remplissage de la grotte du Vallonet", L'Anthropologie, 92: 407-428.

Lumley, H. de; Fournier, A.; Krzepkowska, J., y Echassoux, A. (1988): "L'industrie de Pléistocene inférieur de la grotte du Vallonet, Roquebrune-Cap-Martin, AlpesMaritimes", L'Anthropologie, 92: 501-614.

PeReTto, C. (ed.) (1991): Isernia La Pineta, nuovi contributi scientifici. Isernia, Istituo regionale per gli studi storici del Molise "V. Cuoco".

- (1992): I Primi abitanti della Valle Padana: Monte Poggiolo Nel Quadro delle Conoscenze Europee. Milano: Jaca Book. 
QUEROL, A. (1991): De los primeros seres humanos. Ed. Síntesis. Madrid.

Robers, M. B. (1990): "Amey's Eartham Pit, Boxgrove", In C. TURner (ed.), The Cromer Symposium Norwich 1990, SEQS: field excursion guide book: 62-77. Cambridge: Quaternary Research Association.

Roberts, M. B.; Stringer, C.B.; Parfitt, S.A. (1994): "A hominid tibia from Middle Pleistocene sediments at Boxgrove, UK", Nature, 369: 311-313.

Roebroeks, W.; Conard, N.J., y VAN KOLFSCHOTEN, T. (1992): «Dense forets, cold steppes and the Paleolithic settlement of nothern Europe", Current Anthropology, 33: 551-86.

ROEBROEKS, W., y VAN KOLFSCHOTEN, T. (1994): "The earliest occupation of Europe: a short cronology", Antiquity, 68: 489-503.

ROLLAND, N. (1992): "The Palaeolithic colonization of Europe: an archaeological and biogeographic perspective", Trabajos de Prehistoria, 49: 69-111.

Ruiz Bustos, A.; Toro, I.; Martín, E., y Almohalla, M. (1986): "Procesos evolutivos durante el Cuaternario Medio y Superior en las poblaciones de pequeños mamíferos del sur de la Península Ibérica", Cuadernos de Prehistoria de la Universidad de Granada, 7: 9-35.

SANTONJA, M. (1992): "La adaptación al medio en el Paleolítico Inferior de la Peninsula Ibérica. Elementos para una reflexión". En A. MouRe RomanilLo (ed.), Elefantes, ciervos y ovicaprinos, 37-75. Santander. Universidad de Cantabria.

SANTONJA, M., y VILLA, P. (1990): "The Lower Paleolithic of Spain and Portugal", JournaL OF WORLD PREHISTORY, 4: 45-94.

SCHICK, K.D., y ZHUAN, D. (1993): "Earley Paleolithic of China and Eastern Asia", Evolutionary Anthropology, 2: 22-35.

TEXiER, P.J. (1985): “Chilhac III: Un gisement paléontologique villafranchien soliflué?», Bulletin de la Société Préhistorique Française, 82: 68-70.

Tuffreau, A. (1987): Le Paléolothique inférieur et moyen du Nord de la France (Nord Pasde-Calais, Picardie) dans son cadre stratigraphique. Téshe Doctorat d'Etat Université de Lille. Lille.

TURNER, A. (1982): "Hominids and fellow travellers", South African Journal of Science, 78: 231-237.

- (1992): "Large carnivores and earliest European hominids: changing determinants of resource availability during the Lower and Middle Pleistocene", Journal of Human Evolution, 22: 109-126.

VALOCH, K. (1987): "The Earley Palaeolithic Site Stránská Skála I near Brno (Czecholovakia)", Anthropologie, 25(2): 125-142.

- (1991): "Les premiers peuplements humains en Moravie (Tchécoslovaquie)", In BONIFAY \& VANDEMEERSCH (1991): 189-194.

VILLA, P. (1991): “Middle Pleistocen Prehistory in Southwestern Europe: The state our Knowledge and Ignorance", Journal of Anthropological Research, 47(2): 193-218.

VOLIBRECHT, J. (1992): Das Altpaläolithikum aus den unteren Schichten in Kärlich. Magisterarbeit. Universität Köln, Köln.

WURGES, K. (1986): "Artefakte aus den ältesten Quartärsedimenten (Schichten A-C) der Tongrube Kärlich, Kreis Mayen-Koblenz/Neuwieder Becken", Archäologisches Korrespondenzblatt, 16: 1-6. 